ESAIM: PROCEEDINGS, October 2007, Vol. 22, 122-126

Gabriel Caloz \& Monique Dauge, Editors

\title{
NUMERICAL SIMULATIONS OF FERROMAGNETIC MATERIALS
}

\author{
STÉPHANE LABBÉ ${ }^{1}$
}

\begin{abstract}
In this article, we give a glimpse on recent works in the simulation of ferromagnetic phenomena. We focus here on the computation of the stray field in the periodic case, and the microwave susceptibility which requires the resolution of many ill-conditionned linear systems.

Résumé. Dans cet article, nous présentons des travaux récents concernant la simulation numérique de phénomènes ferromagnétiques. Nous nous concentrons sur le calcul du champ démagnétisant avec des conditions périodiques, et celui de la susceptibilité hyperfréquence qui nécessite la résolution de nombreux systèmes linéaires mal conditionnés.
\end{abstract}

\section{INTRODUCTION}

Technological applications of ferromagnetic materials are nowadays numerous among which one finds nano electronic devices, telecommunication or magnetic data recording. In all these fields, the numerical simulation plays a big role, and in order to confront the numerical results to the experiments, the microwave susceptibility, which measures the response of the sample to an external periodic magnetic excitation, is a possible tool.

The model usually used for modelizing ferromagnetic materials is the micromagnetism, introduced by W.F. Brown in 60'. This model, based upon a thermodynamic analysis of the ferromagnetic phenomenon, is quickly recalled in section 1 (see also $[1,3,5,6]$ ). In a second section, we focus on the discretization of the stray field (more details are given in $[4,8]$ while its use is described in $[7,10]$ ). The last part is dedicated to the microwave susceptibility computation [9] and particularly to the strategy adopted to treat the ill-conditioned systems that need to be solved for each frequence.

\section{A MODEL FOR FERROMAGNETiC MATERIALS: THE MiCROMAGNETiSM}

Let $\Omega$ be an open bounded set of $\mathbb{R}^{3}$, we designate by $m$ in $H^{1}\left(\Omega, \mathbb{S}^{2}\right)$ the magnetization (here, $\mathbb{S}^{2}=\{x \in$ $\left.\mathbb{R}^{3}|| x \mid=1\right\}$ ). The energy functional is given by:

$$
E(m)=A \int_{\Omega}|\nabla m|^{2} d x+\int_{\mathbb{R}}\left|h_{\mathrm{d}}(m)\right|^{2} d x+\int_{\Omega} \phi(m) d x-2 \int_{\Omega} h_{\mathrm{ext}} \cdot m d x .
$$

The first term is the exchange contribution and takes into account microscopic spin-spin interactions on the crystal network. The last three terms modelize respectively the stray energy $\left(h_{\mathrm{d}}(m)\right.$ is the stray field), anisotropy and external energies (see [1] for a more detailed description). Minimizers of this energy are physically relevant configurations.

\footnotetext{
${ }^{1}$ Laboratoire de Mathématiques, Bât. 425, Université Paris-Sud 11, 91405 Orsay cedex.
} 
As far as dynamical effects are concerned, one usually uses the Landau-Lifchitz equations that we write on purpose:

$$
\frac{\partial m}{\partial t}=-m \wedge\left(h_{\mathrm{tot}}(m)+h_{\mathrm{ext}}\right)-\alpha m \wedge\left(m \wedge\left(h_{\mathrm{tot}}(m)+h_{\mathrm{ext}}\right)\right),
$$

where $h_{\text {tot }}(m)=A \triangle m+h_{\mathrm{d}}(m)+\nabla_{m} \phi(m)$, and $h_{\text {tot }}(m)+h_{\text {ext }}$ is the effective field. This dynamical system preserves the magnitude of the magnetization $m$ and dissipates the energy in the case where the external field does not depend on time. At least formally, one expects from these properties that solutions of (1) converge to minimizers of $E(m)$ under the constraint $m \in H^{1}\left(\Omega, \mathbb{S}^{2}\right)$.

\section{THE STRAY FIELD}

The design of a numerical scheme for the simpulation of (1) can be easily obtained by a finite difference discretization in time and a finite volumes discretization in space on a regular mesh. The only difficulty is the computation of the stray field which is obtained via the formula:

$$
h_{\mathrm{d}}(m)=\operatorname{grad} \operatorname{div} \triangle^{-1} \tilde{m} .
$$

(Here $\tilde{m}$ stands for the extension by 0 of $m$ on $\mathbb{R}^{3}$ ). We propose to compute the stray field with a finite volume like scheme. Let $u \in L^{2}(\Omega)$ and $\left(\omega_{i}\right)_{i \in\{1, \ldots, N\}}$ a regular cubic mesh of $\Omega$. We define

$$
\begin{aligned}
P: L^{2}(\Omega) & \rightarrow L^{2}(\Omega) \\
u & \mapsto P(u)=\sum_{i \in\{1, \ldots, N\}}\left(\frac{1}{\left|\omega_{i}\right|} \int_{\omega_{i}} u(x) d x\right) \chi_{i},
\end{aligned}
$$

the $L^{2}$ orthogonal projection on piecewise constant functions. Here, $\chi_{i}$ stands for the characteristic function of $\omega_{i}$. Denoting by $P^{*}$ the canonical injection of the piecewise functions into $L^{2}(\Omega)$, the discrete stray field operator $h_{\mathrm{d}, h}$ is defined by

$$
h_{\mathrm{d}, h}=P \circ h_{\mathrm{d}} \circ P * .
$$

This approximation can be shown to be convergent and the spectrum of the discrete operator preserves the properties of positivity and contraction of the continuous one [8]. Using the regular structure of the mesh, it is easily seen that the matrix associated to the discrete operator has a block-Toeplitz structure. Although it is completely dense, this enables to reduce the required storage to $O(N)$, while the evaluation of the stray field (which is a matrix-vector product) can be done with a complexity $O(N \log (N))$. Recent developments have generalized this approach to domains that are periodic in one or two space directions.

Indeed, using a multi-level mesh, the contribution on the main period is computed with the maximal accuracy using the fast method developed in the case of a finite domains. Then, using the scale independance of the demagnetizing operator, we compute a coarser approximation of the stray field on a mesh whose cells ares twice bigger in each periodic direction. The process is repeated several times and the combination of the approximations on each level gives an approximation of the total field on the whole space (see fig. 1). The main advantage of the method is that we compute the contribution of the stray field on each level, from the more accurate to the coarser, with the same fast method, and the same matrix (see fig. 2 for the convergence rate and [4] for details).

The other terms in the equation are classical, and we use for the time discretization an explicit finite difference scheme in which the time step is optimized in order to ensure the convergence of the scheme and an optimal decreasing of the energy $[7,10]$. 


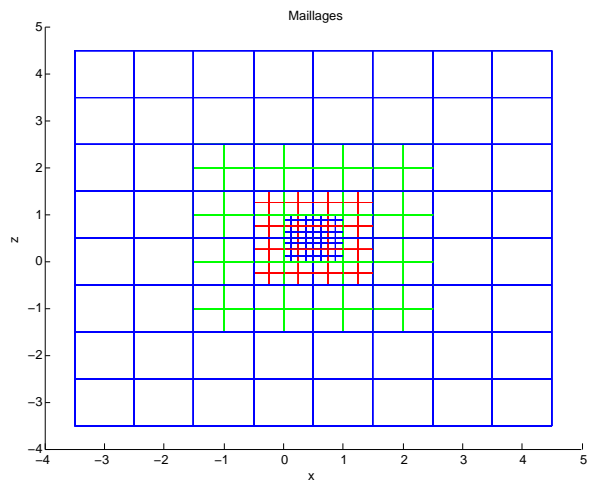

Figure 1. Dyadic mesh for a periodic domain in two directions $(8 \times 8 \times 8$ mesh of the core domain).

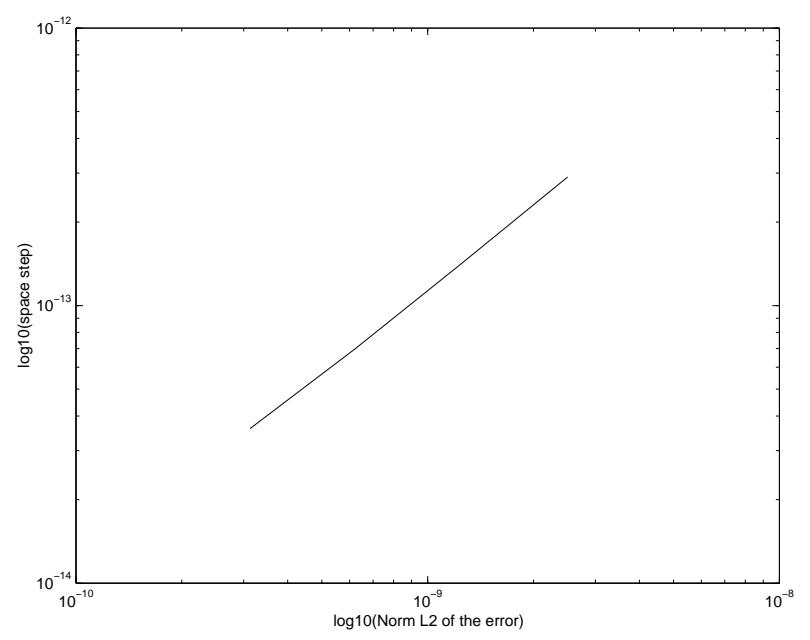

FiguRE 2. Accuracy of the multilevel algorithm.

\section{MicRowaVe SUSCEPTIBILITY}

A possible tool used in order to compare three dimensional simulations to experiments is the microwave susceptibility. The main idea is to light a sample with a monochromatic magnetic wave, in the three space 
directions of space, and retrieve the energy scattered by the sample. Mathematically, the problem is written as follows: given an equilibrium state $m_{\text {eq }}$, and $\left(h_{1}, h_{2}, h_{3}\right)$ an orthogonal basis of $\mathbb{R}^{3}$, find for each frequency $\omega \in \mathbb{R}_{*}^{+}$and $j=\{1,2,3\}$, the vector fields $\delta m_{j} \in L^{2}\left(\Omega, \mathbb{R}^{3}\right)$, such that $m_{\mathrm{eq}}+\delta m_{j} e^{i \omega t}$ is the solution of the linearization of (1) (first order in $h_{j}, \delta m_{j}$ ) for $h_{\mathrm{ext}}=h_{j} e^{i \omega t}$ around $m_{\mathrm{eq}}$.

The $3 \times 3$ complex matrix $\chi$ defined by

$$
\chi h=\frac{1}{|\Omega|} \int_{\Omega} \delta m,
$$

where $h={ }^{t}\left(h_{1}, h_{2}, h_{3}\right)$ and $\delta m={ }^{t}\left(\delta m_{1}, \delta m_{2}, \delta m_{3}\right)$ is called the microwave susceptibility.

The linearization of (1) around $m_{\mathrm{eq}}$ leads to an equation that can be written as

$$
i \omega \delta m_{j}-\left(D_{1}\left(m_{\mathrm{eq}}\right) \circ h_{\mathrm{tot}}+D_{2}\left(m_{\mathrm{eq}}\right)\right) m_{j}=D_{1}\left(m_{\mathrm{eq}}\right) h_{j},
$$

where, for every $v \in \mathbb{R}^{3}$

$$
\begin{aligned}
& D_{1}\left(m_{\mathrm{eq}}\right) v=-m_{\mathrm{eq}} \wedge v-\alpha m_{\mathrm{eq}} \wedge\left(m_{\mathrm{eq}} \wedge v\right), \\
& D_{2}\left(m_{\mathrm{eq}}\right) v=h_{\mathrm{tot}}\left(m_{\mathrm{eq}}\right) \wedge v-\alpha m_{\mathrm{eq}} \wedge\left(v \wedge h_{\mathrm{tot}}\left(m_{\mathrm{eq}}\right)\right) .
\end{aligned}
$$

In order to compute the discretized microwave susceptibility, we replace in (3) the continuous version of the operators by their discretized ones. Relevant equilibrium states, $m_{\mathrm{eq}, h}$ are obtained by computing time limits of dynamical solutions. The linear system that needs to be solved rewrites (the frequency $\omega$ being given)

$$
(i \omega I d-M) m=h,
$$

where $h$ and $m$ are vectors of $\mathbb{R}^{3 N}, I d$ is the identity matrix on $\mathbb{R}^{3 N}$ and $M$ is a square matrix of order $3 N$. The matrix $M$ is given by

$$
M=D_{1}\left(m_{\mathrm{eq}, h}\right) \circ h_{\mathrm{tot}, \mathrm{h}}+D_{2}\left(m_{\mathrm{eq}, h}\right) .
$$

It turns out that the system (4) is very ill-conditionned, and there is a crucial need for a suitable preconditioner. Such a preconditioning strategy is proposed in [9] where it is shown that the matrix $M$ can be efficiently preconditioned by $\left(h_{\mathrm{tot}, \mathrm{h}}\right)^{-1}$. The cost of the inversion of the full operator $h_{\mathrm{tot}, \mathrm{h}}$ being too high, we use an approximation $\tilde{h}_{\mathrm{tot}, \mathrm{h}}$ of this operator obtained as the projection of $h_{\mathrm{tot}, \mathrm{h}}$ on skew matrices in the sense of the Froebenius norm.

The improvement of the convergence obtained using this preconditioning method (see fig. 3, for the exchange case), already permits to compute the susceptibility on large meshes (see [2]).

\section{REFERENCES}

[1] F. Alouges. Mathematical models in micromagnetism. in this volume.

[2] F. Boust, N. Vukadinovic, and S. Labbé. Domain wall resonant modes in nanodots with a perpendicular anisotropy. Journal of Magnetism and Magnetic Materials, 272-276:708-710, 2004.

[3] W.F. Brown. Micromagnetics. Interscience Publishers, 1963.

[4] S. Faure and S. Labbé. Multi-levels computation of the demagnetization field in periodic domains applied to micromagnetism computations. Technical report, Université Paris-Sud 11, Laboratoire de Mathématique, 2006.

[5] L. Halpern and S. Labbé. La théorie du micromagnétisme. Modélisation et simulation du comportement des matériaux magnétiques. Matapli, (66):77-92, 2001.

[6] A. Hubert and R. Schäfer. Magnetic Domains : The Analysis of Magnetic Microstructures. Springer-Verglag, 2000.

[7] S. Labbé. Simulation numérique du comportement hyperfréquence des matériaux ferromagnétiques. PhD thesis, Université Paris 13, Décembre 1998.

[8] S. Labbé. Fast computation for large magnetostatic systems adapted for micromagnetism. SISC SIAM Journal on Scientific Computing, 26(6):2160-2175, 2005.

[9] S. Labbé. A preconditioning strategy for microwave susceptibility in ferromagnets. AES, Advances in Engineering Software, to appear, 2006.

[10] S. Labbé and P.-Y. Bertin. Microwve polarisability of ferrite particles with non-uniform magnetization. Journal of Magnetism and Magnetic Materials, 206:93-105, 1999. 

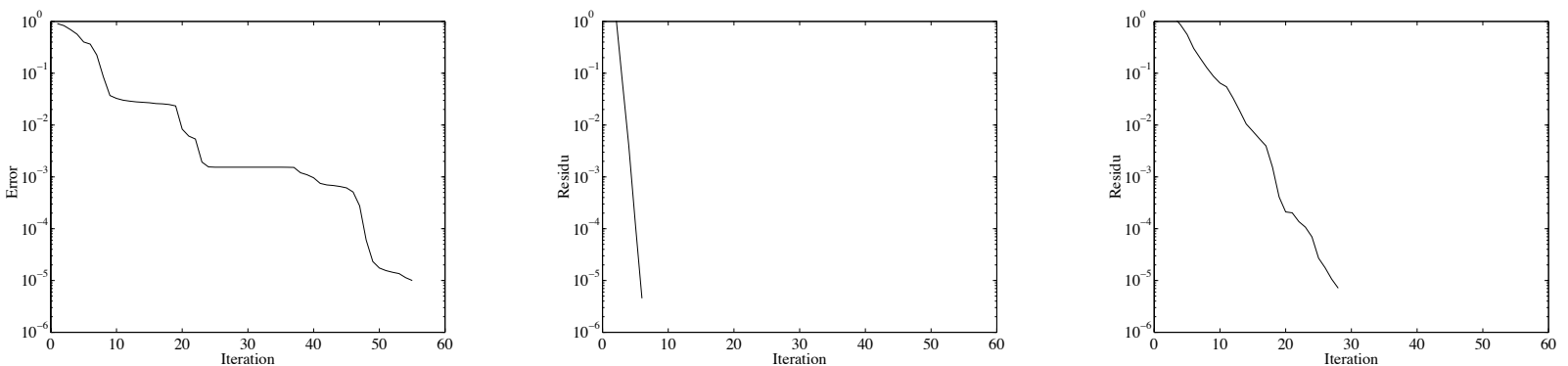

FiguRE 3. Example of convergence curves for non preconditioned system, preconditioned system and approached preconditioned system. 